\title{
Preliminary Sizing of a Medium Range Blended Wing-Body using a Multidisciplinary Design Analysis Approach
}

\author{
Alessandro Sgueglia ${ }^{1,2^{*}}$, Peter Schmollgruber ${ }^{1}$, Emmanuel Benard ${ }^{2}$, Nathalie Bartoli ${ }^{1}$ and \\ Joseph Morlier ${ }^{2}$ \\ ${ }^{1}$ ONERA/DTIS - The Aerospace French Lab, 2 avenue Edouard Belin - 31055 Toulouse (France) \\ ${ }^{2}$ ISAE-Supaero, 10 avenue Edouard Belin, 31055 Toulouse (France)
}

\begin{abstract}
The aviation's goal for the next decades is to drastically reduce emissions, but to achieve this goal a breakdown in aircraft design have to be considered. One of the most promising concept is the Blended WingBody, which integrates aerodynamics, propulsion and structure, and has a better aerodynamics efficiency, thanks to the reduction of the wetted surfaces. In this work the feasibility of a short/medium range BWB with 150 passengers (A320 type aircraft, Entry Into Service 2035) is studied, considering different disciplines into the sizing process. To supply the lack of reference data, an approach that goes from high fidelity to validate low fidelity models has been set up. Also certification aspects have been taken into account in an off-design analysis. To evaluate the advantages of the proposed concept, it has been compared with an aircraft of the same class, the A320 Neo, resized to match the EIS2035 hypothesis: results show that the BWB is a concept that shows a gain in fuel consumption, especially on longer ranges.
\end{abstract}

\section{Introduction}

One of the aviation's goals for the next decades is to lower its environmental footprint. The classical "tube and wing" configuration has been developed over the past 50 years, and it has still few potential gains: a breakdown at the conceptual level is needed. Among all the possible configurations, the Blended Wing-Body (BWB) [1-3] is one of the most promising: the integration of payload, control surfaces and even propulsion into the wing body provides better aerodynamic performances than those of conventional aircrafts. However these advantages are counterbalanced by several drawbacks such as its instability and the more complex architecture. The goal of this work is to present a procedure to design, at conceptual level, a Blended Wing-Body, using the tool called FAST [4], developed by ONERA and ISAE-Supaero. Mainly low fidelity methods are used in preliminary design: since the Blended Wing-Body is an unconventional aircraft, the methods used need to be checked for the new configuration. Due to the lack of reference

* Corresponding author: alessandro.sgueglia@onera.fr 
data, high fidelity methods (like Computational Fluid Dynamics or Finite Element Method) has been used as reference to validate and eventually correct the low fidelity. To assess the validity of methods, a BWB reference geometry has been designed at ONERA and ISAESupaero [5]. Certification constraints will be also verified to ensure the validity of the proposed concept. In the next sections will be first presented the modifications to the design process, then the results on the design mission and the performances on operational ranges will be shown; finally certification aspects will be considered in an off design analysis.

\section{Methodology}

\subsection{FAST description}

The main tool used in this work is the sizing tool FAST [4]. It is a Multidisciplinary Design Analysis tool, which takes into account different disciplines (aerodynamics, propulsion, structures and perfomances) and uses low fidelity methods (engineering methods or semiempirical equations, mainly based on the handbook written by AIRBUS and ISAE-Supaero [6]). In Figure 1 is shown the general scheme of the code, using the XDSM standard [7].

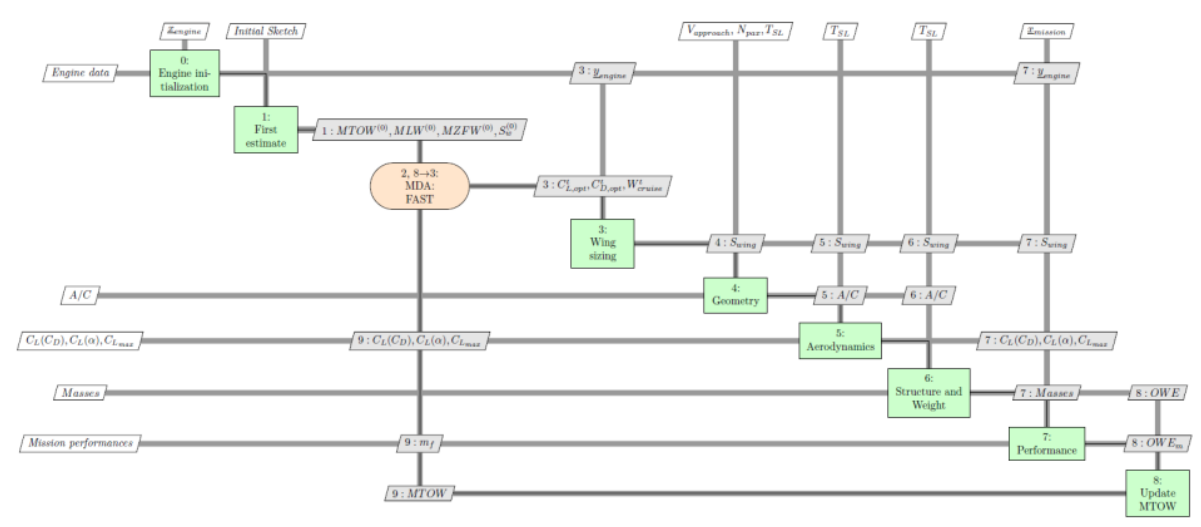

Fig. 1. FAST's xDSM scheme

In order to consider a Blended Wing-Body the design process shown in Figure 1 has to be changed in some parts: the first estimation needs to be reviewed, then the geometry model has to be modified to consider the absence of the fuselage, replaced by a centerbody that can be schematized as a wing with a greater thickness to chord ratio; finally the aerodynamics and the mass models have to be reviewed, to correct the methods used in FAST. Due to the lack of reference open source data, a numerical approach has been used: for the aerodynamics the code $\mathrm{SU}^{2}$ [8] has been used to quantify the difference using the methods coded in FAST, meanwhile the free code OpenNastran [9] has been used to estimate the weight of the centerbody. The common geometry from ISAE-Supaero and ONERA has been used for the validation [5]: it is shown in Figure 2, meanwhile in Table 1 the Top Level Aircraft Requirements and the main geometric parameters are reported. The TLAR are similar to the A320 Neo type aircraft (medium range aircraft with 150 passengers). 


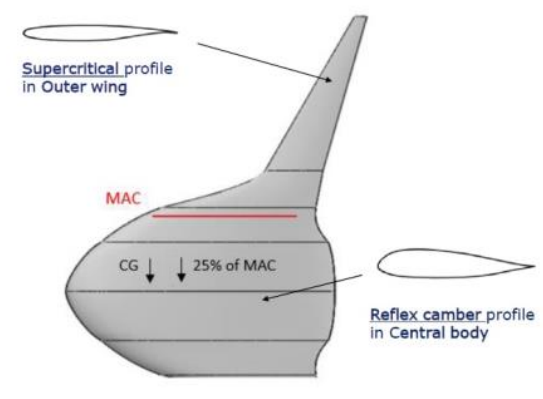

Fig. 2. Common Blended Wing-Body reference geometry
Table 1. TLAR and geometric parameters for the BWB reference geometry

\begin{tabular}{|l|rl|}
\hline Number of passengers & 150 & \\
Cruise Mach number & 0.78 & \\
Range & 2750 & $\mathrm{NM}$ \\
\hline MTOW & 80.2 & $\mathrm{t}$ \\
Wing span & 41 & $\mathrm{~m}$ \\
Wing surface & 313 & $\mathrm{~m}^{2}$ \\
\hline
\end{tabular}

\subsection{Constraint analysis}

The wing area is determined in order to produce enough lift in approach condition (at the maximum lift coefficient) and have volume enough to store all the fuel needed for the mission. In the case of short and medium range aircraft (that is the case of the ISAESupaero and BWB geometry) the first criteria is the most stringent one, meanwhile the second criteria becomes relevant in long and very long range aircraft. Knowing the value of maximum CL, with flaps and slats deflected, it is possible to compute the wing area starting from the lift coefficient. In the case of a Blended Wing-Body, in which the whole structure is a lifting body, the lift equation approach underestimates the area, so the necessity to find a new criterion to size the wing arises. In order to find the proper way, a constraint analysis $[10,11]$ has been performed, giving in output the constraint diagram for the new proposed concept. The constraint diagram is the plot of the thrust loading (thrust at sea level over the weight) with respect to the wing loading (weight over wing surface). The criteria considered are:

- Approach condition: the aircraft has to be able to produce enough lift in approach condition.

- Takeoff constraint: the aircraft needs to takeoff in a certain length.

- Initial climb constraint: at the beginning of the climb, the aircraft has to be able to climb with a climb rate of at least $2.4 \%$ with one engine inoperative.

- Top of climb constraint: at the top of climb (beginning of cruise) the aircraft needs to have a reserve of vertical speed of $300 \mathrm{ft} / \mathrm{min}$.

- Geometry constraint: specific for the Blended Wing-Body, ensure that the wing area is not lower than the central body area, which is sized in order to allocate all the passengers.

Models used to build the diagram are semi-empirical and engineering models [11], so a certain level of uncertainty needs to be taken into account to analyse the results. The constraint diagram is shown in Figure 3: both the wing and the thrust loading are referred to the Maximum Takeoff Weight (MTOW). The feasible domain is the area above the takeoff, initial climb and top of climb condition, and on the left of geometric and landing 
conditions. From the plot is possible to note that the approach condition is not anymore the most stringent condition, since the area required is lower than that of the centerbody (which is fixed by the number of passengers to be allocated), but it is the top of climb condition. It is also possible to note the presence of a minimum point, which minimize both thrust and wing area.

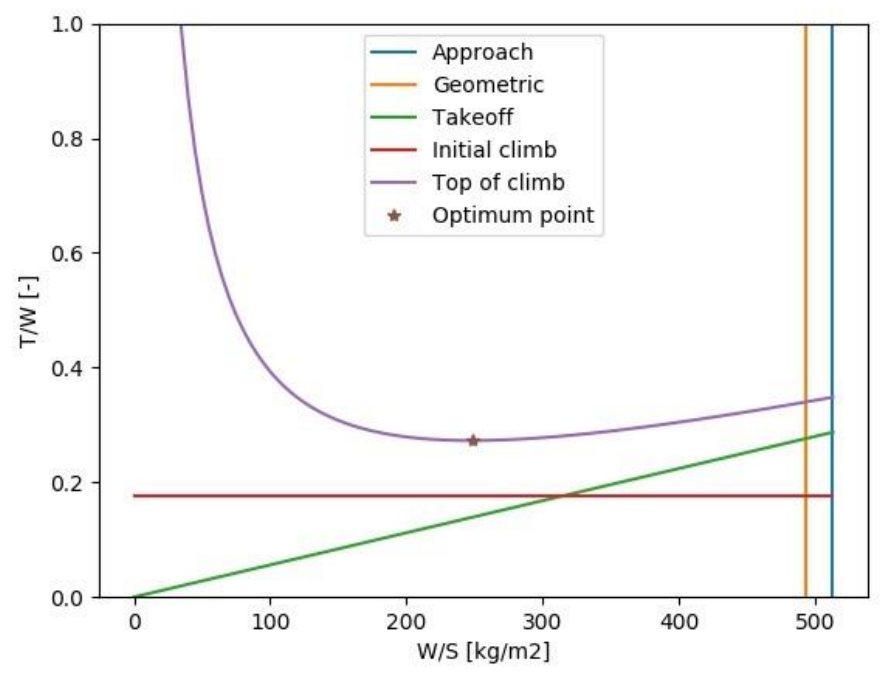

Fig. 3. Constraint diagram for the Blended Wing-Body. Reference weight for the thrust and the wing loading is the MTOW; the reference thrust is the maximum thrust at sea level

\subsection{Aerodynamics model}

The aerodynamics model in FAST is based on semi-empirical equations that a priori are valid only for a classical tube and wing configuration. In the Blended Wing-Body the fuselage is not tubular anymore but it is treated as a wing, with higher thickness to chord ratio. Cerquetani studied the aerodynamics of the reference geometry [5], using an eulerian approach (thus neglecting viscosity) with a Mach number of 0.78 and comparing the results with the models used in FAST, as shown in Figure 4 (left). The code used in this code is $\mathrm{SU}^{2}$ [8], a free CFD software, developed at the Stanford University. With these results, it has been possible to use some corrective factors to make the two curves match (Figure 4, right). The assumption that the correction is valid to different geometries has been made. This is a strong assumption, which needs to be refined in the future studying different geometries and including also the effect of the viscosity.

\subsection{Mass model}

The last modification concerns the mass model: in the BWB a central cabin which is a wing section (with a bigger thickness to chord ratio) takes the place of the classical tubular fuselage. In order to estimate the weight of the centerbody, a Finite Element Method (FEM) analysis has been used in this section, using the open software OpenNastran [9]. In the reference geometry, the centerbody has been sized in order to allocate 18 passengers per 
row in 3 bays, following the indications reported in the work of Bradley [2]. It has been chosen an integrated stiffened skin concept, with bubbles to withstand the pressurization loads, with a single spar at the $70 \%$ of the total length. The material is the Aluminium Alloy 7075 , typically used for aeronautical structures. The applied loads have also been suggested by the work of Bradley [2]. The resulting weight is of about 22 tons.
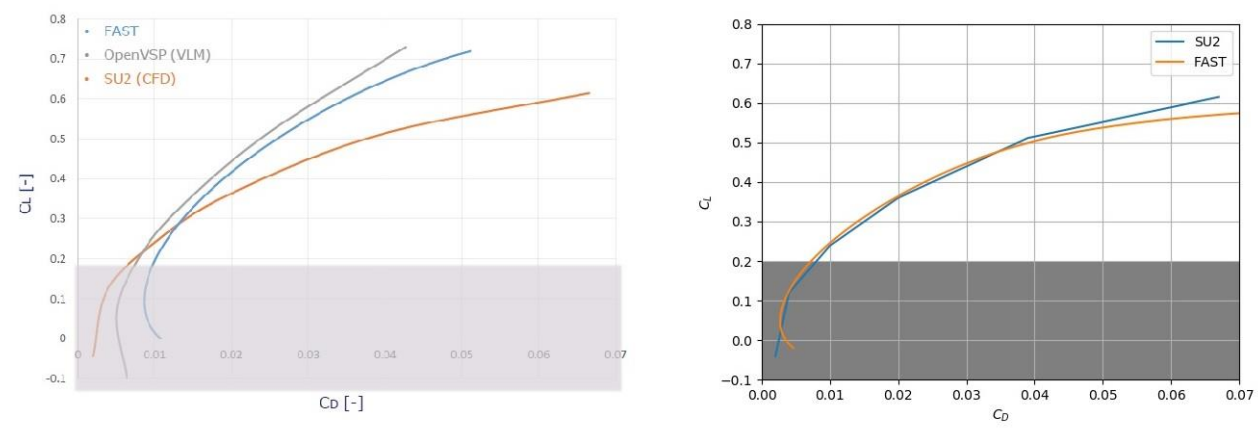

Fig. 4. Comparison between the polars computed using $\mathrm{SU}^{2}$, FAST and the VLM method on the BWB reference geometry (on the left) and between $\mathrm{SU}^{2}$ and FAST, with the application of corrective factors on the latter (on the right)

\section{Results}

\subsection{Design loop}

In this section results for the Blended Wing-Body have been presented. The TLAR are reported in Table 3: differently from previous works on the BWB, in this work the focus is on a short-medium range BWB, with a number of passengers of 150 (instead of 400 or above). The span is limited by airports' constraint. The hypothesis of Entry Into Service 2035 has been made: wing, landing gear and tails have a reduced weight due to the use of innovative materials, as reported by Sgueglia et al. [12] As reference the A320 Neo (resized to match the EIS2035 hypotheses) has been considered. An engine with high By-Pass Ratio has been used $(\mathrm{BPR}=10)$, in line with the engine mounted on the A320 Neo [13].

Table 2. Top Level Aircraft Requirements used to design the BWB concept and the A320Neo.

\begin{tabular}{|l|rc|}
\hline Range & 2750 & NM \\
Number of passengers & 150 & \\
Mach number & 0.78 & \\
Max span & 36 & $\mathrm{~m}$ \\
\hline
\end{tabular}

In order to match the maximum span limit, the centerbody has been resized and is stricter than the reference aircraft, thus the estimation presented earlier is very conservative. Three different scenarios have been considered, to study the effect of the centerbody weight: they are reported in Table 4 . 
Table 3. Scenarios considered for the centerbody weight: the worst case is the most conservative, corresponding to the estimation of $22 \mathrm{t}$; for the other cases a reduction with respect to this estimation has been considered.

\begin{tabular}{|r|r|l|}
\hline Best & Medium & Worst \\
\hline$-40 \%$ & $-20 \%$ & 0 \\
\hline
\end{tabular}

In Table 5 is reported the comparison between the A320 Neo and the three BWB baselines: in all the cases the Maximum Takeoff Weight (MTOW) and the Operating Weight Empty (OWE) is bigger than that of the reference aircraft, mainly due to the centerbody structure: since it is a more complex concept, that requires reinforcements to carry out the pressurisation and the bending moment of the outer wing, it is heavier than a tubular fuselage. Even if the BWB is heavier, the mission fuel is reduced of about $18 \%$ in the best case and $6 \%$ in the worst case: this effect is due to the fact that the BWB is aerodynamically more efficient (with a maximum L/D of 23 instead of 17) thanks to the reduction of wetted area. Another aspect to be noted is that the cruise altitude is bigger for a BWB: the cruise starts at the point of maximum aerodynamic efficiency, but since the wing area is more than doubled, the aircraft requires to climb more to reach this condition.

Table 4. Comparison between the A320Neo and the three different BWB baselines

\begin{tabular}{|ll|r|r|r|r|}
\cline { 3 - 6 } \multicolumn{1}{l|}{} & & A320Neo & BWB, worst & BWB, medium & BWB, best \\
MTOW & {$[\mathrm{t}]$} & 67.8 & 80.6 & 74.2 & 68.5 \\
OWE & {$[\mathrm{t}]$} & 38.6 & 42.2 & 47.1 & 52.4 \\
Wing area & {$\left[\mathrm{m}^{2}\right]$} & 115.7 & 340.1 & 341.6 & 349 \\
Max L/D & {$[-]$} & 17.2 & 23.7 & 23.6 & 23.4 \\
Cruise altitude & {$[\mathrm{kft}]$} & 35.7 & 42.4 & 40.8 & 39.5 \\
Mission fuel & {$[\mathrm{t}]$} & 15.6 & 12.7 & 13.5 & 14.6 \\
\hline
\end{tabular}

\subsection{Operational mission performances}

Finally, once the aircraft has been sized with the TLAR reported in Table 3, some analysis to study the fuel consumption on different operational ranges have been studied: in fact an aircraft is not always flying at is design range, but according to the necessity of the airlines it can fly on routes with different ranges. The operational ranges vary between 800 and $1600 \mathrm{NM}$. The fuel consumption of the reference aircraft and the three BWB considered with respect to the operational range is shown in Figure 5. These results show that the Blended Wing-Body is not efficient for small ranges: there is a point starting from which it becomes more advantageous than the traditional aircraft, and this point is more shifted to the right when the centerdoby weight increases. This happens because the BWB has better aerodynamics performances in cruise, but it is less efficient during the climb and the descent phase; also, as already noted in Table 5, the cruise altitude is bigger, that is the BWB needs to climb more and so spend more fuel. In conclusion, the longer is the cruise segment, the more the BWB is advantageous. This is in line with the previous works [1-3] that considered only long and very long range for the BWB: it is on these routes that the $\mathrm{BWB}$ is most performing. 


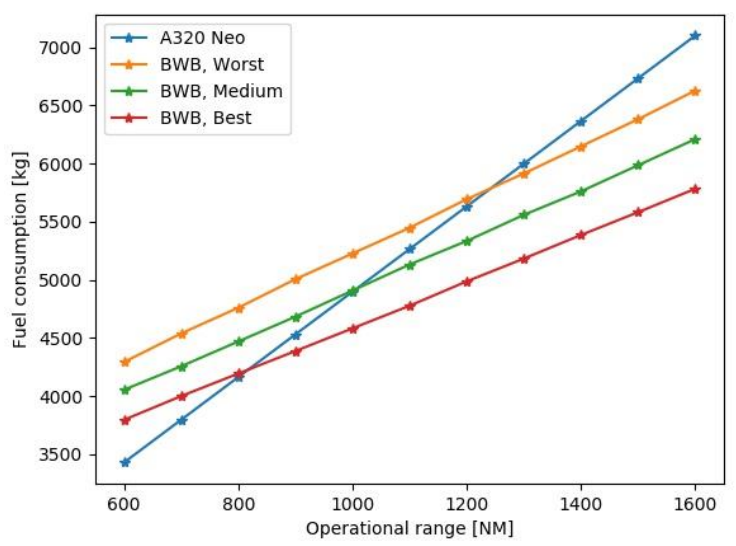

Fig. 5. Fuel consumptions with respect to operational ranges for the reference aircraft and the three BWB considered.

\subsection{Off-design certification analysis}

Finally, an off-design certification analysis has been carried out. Certifications play a key role during the design process: in fact an aircraft should not only be feasible, but should also respect the certifications. For this reason an off design analysis of the certification for the unconventional aircraft here considered has been carried out. The module developed by Schmollgruber et al. and implemented in FAST has been used. The certifications here considered are the CAT.POL [14] to define the en route performances and the CS-25 [15] for large aircrafts for the takeoff and approach performances. They are detailed below:

- CAT.POL.A.410(a) [14]: this certification requires that at the top of climb (1) and top of descent (2) the aircraft has a reserve of vertical speed of $300 \mathrm{ft} / \mathrm{min}$.

- CS-25.119(a) [15]: this certification requires that, in landing condition and with all the engines operating, the steady gradient flight may not be less than $3.2 \%$.

- CS-25.121(a) [15]: this certification requires that, in takeoff condition with the landing gears extracted and one engine inoperative, the steady gradient of climb must be positive.

- CS-25.121(b) [15]: this certification requires that, in takeoff condition and one engine inoperative, the steady gradient of climb, at the flight path point when the landing gears are fully retracted (400ft), may not be less than $2.4 \%$.

- CS-25.121(c) [15]: this certification requires that, in the en-route configuration at the end of takeoff with one engine inoperative, the steady gradient of climb may not be less than $1.2 \%$.

- CS-25.121(d) [15]: this certification requires that, in approach condition with all the engines operating, the steady gradient of climb may not be less than $2.1 \%$.

In Table 6 are reported performances at the flight path points required by the certifications above described for the three Blended Wing-Body concepts here considered, and the minimum value required. It can be seen that the certifications are always respected, and that the most stringent (in percentage) is the top of climb condition: this is in line with the logic used to size the wing, since the design has been made in order to have the reserve of vertical speed at that point. 
Table 5. Comparison between the performances at the flight path points required by certifications and the minimum value for the three BWB concepts considered.

\begin{tabular}{|ll|l|r|r|r|}
\cline { 3 - 6 } \multicolumn{1}{c|}{} & \multicolumn{2}{l|}{$\begin{array}{l}\text { Required min. } \\
\text { value }\end{array}$} & $\begin{array}{l}\text { BWB, } \\
\text { worst }\end{array}$ & $\begin{array}{l}\text { BWB, } \\
\text { medium }\end{array}$ & $\begin{array}{l}\text { BWB, } \\
\text { best }\end{array}$ \\
\hline CAT.POL.A.410(a) & {$[\mathrm{ft} / \mathrm{min}]$} & 300 & 366.6 & 353.4 & 367.9 \\
CAT.POL.A.410(b) & {$[\mathrm{ft} / \mathrm{min}]$} & 300 & 361.3 & 349.4 & 363.6 \\
CS-25.119(a) & {$[\%]$} & 3.2 & 9.24 & 10.8 & 13.3 \\
CS-25.121(a) & {$[\%]$} & 0 & 0.27 & 0.34 & 0.55 \\
CS-25.121(b) & {$[\%]$} & 2.4 & 2.56 & 2.74 & 3.76 \\
CS-25.121(c) & {$[\%]$} & 1.2 & 5.07 & 5.51 & 6.24 \\
CS-25.121(d) & {$[\%]$} & 2.1 & 2.33 & 2.91 & 3.24 \\
\hline
\end{tabular}

\section{Conclusions and perspectives}

In this work the feasibility of a short/medium range Blended Wing-Body, for 150 passengers (EIS2035) has been studied. The tool used is FAST, developed by ONERA and ISAE-Supaero. Firstly the design process has been reviewed, to consider the unconventional geometry: modifications in the geometry, aerodynamics and mass model have been described. In particular, as shown from the constraints analysis, the most stringent condition to size the wing area is the top of climb, differently from conventional configurations. Then, results are presented, compared with the A320 Neo, aircraft of the same type as the BWB here considered. Three different baselines, varying the centerbody weight, have been considered. Results show that, even if the Blended Wing-Body has the disadvantage to be heavier, there is a gain of fuel (from 6 to 20\%) thanks to the better lift to drag ratio. On operational missions, results show that the BWB concept is more advantageous starting from a certain range: in fact the main gain for a BWB is in the cruise segment, meanwhile in other segments is less efficient than the conventional configuration; then longer is the range, better is the concept. Finally an off-design analysis has been carried out: showing that all the design constraint here considered are satisfied, and that the top of climb is the most stringent. In the future, better refinements of the aerodynamics and the mass models have to be carried out, also considering different configurations and methods (like the RANS for the aerodynamics). Aero-propulsive integration has to be also taken into account, considering the integration between nacelle and structure with innovative propulsive chains (like the distributed hybrid propulsion) to better assess the advantages of this concept in the chosen technology horizon.

\section{References}

1. R.H. Liebeck, Design of the Blended Wing-Body Subsonic Transport, Journal of Aircraft, 41, 1 (2004)

2. K.R. Bradley, A Sizing Methodology for the Conceptual Design of Blended WingBody Transports, NASA/CR-2004-213016 (2004)

3. M. Brown and R. Vos, Conceptual Design and Evaluation of Blended Wing-Body Aircraft, AIAA SciTech Meeting (2018)

4. P. Schmollgruber, J. Bedouet, A. Sgueglia, S. Defoort, R. Lafage, N. Bartoli, Y. Gourinat and E. Benard, Use of a Certification Constraints Module for Aircraft Design Activities, AIAA Aviation Forum (2017)

5. L. Cerquetani, High Fidelity Aerodynamics Models for Blended Wing-Body Design, oral presentation, ICAA (2018) 
6. W.P. Dupont and C. Colongo, Preliminary Design of a Commercial Transport Aircraft, class notes, ISAE-Supaero\&Airbus, English edition (2014)

7. A.B. Lambe and J.R.R.A. Martins, Extensions to the Design Structure Matrix for the Description of Multidiplinary Design, Analysis, and Optimization Processes, Structural and Multidisciplinary Optimization, 45, 2, 273-284 (2012)

8. F. Palacios, T.D. Economon, A.C. Aranake, S.R. Copeland, A.K. Lonkar, T.W. Lukaczyk, D.E. Manosalvas, K.R. Naik, A. Santiago Padron, B. Tracey, A. Variyar and J.J. Alonso, Stanford University Unstructured $\left(S U^{2}\right)$ : Open-source Analysis and Design Technology for Turbulent Flows, AIAA SciTech Meeting (2014)

9. OpenNASTRAN software, https://github.com/nasa/NASTRAN-95

10. D.P. Raymer, Aircraft Design: A Conceptual Approach (AIAA Education Series, 2nd ed., 1989)

11. J. Roskam, Airplane Design - Part I (DAR Corporation, 1st ed., 1989)

12. A. Sgueglia, P. Schmollgruber, N. Bartoli, O. Atinault, E. Benard, J. Morlier, Exploration and Sizing of a Large Passenger Aircraft with Distributed Electric Ducted Fans, AIAA SciTech Meeting (2018)

13. LEAP engine, https://www.cfmaeroengines.com/engines/leap/

14. Official Journal of the European Commission, Commission Regulation (EU) $n$. 965/2012 (2012)

15. EASA, Certification Specification for Large Aeroplanes CS-25, Amd. 3 (2017) 